\title{
On certain infinite integrals involving Struve functions and parabolic cylinder functions
}

\author{
By S. C. Mrtra.
}

(Received 28th May, 1945. Read 2nd June, 1945.)

(Revised manuscript received 12th February, 1946.)

The object of the present note is to obtain a number of infinite integrals involving Struve functions and parabolic cylinder functions.

1. G. N. Watson ${ }^{(1)}$ has proved that

$$
\int_{0}^{\infty} e^{-t x^{2}} x^{m} D_{n}(x) d x=\frac{\sqrt{\pi} 2^{t(n-m-1)} \Gamma(m+1)}{\Gamma\left(\frac{1}{2} m-\frac{1}{2} n+1\right)} \quad(R(m)>-1) .
$$

From (1)

$$
\begin{aligned}
& \int_{0}^{\infty} e^{-4 x^{2}} x^{m}{ }_{p} F_{q}\left(a_{1}, \ldots, a_{p} ; c_{1}, \ldots, c_{q} ; x^{2} y^{2}\right) D_{n}(x) d x \\
= & \frac{\sqrt{\pi} 2^{2(n-m-} \Gamma(m+1)}{\Gamma\left(\frac{1}{2} m-\frac{1}{2} n+1\right)}{ }_{p+2} F_{q+1}\left[\begin{array}{l}
a_{1}, \ldots, a_{p}, \frac{1}{2} m+\frac{1}{2}, \frac{1}{2} m+1 ; \\
c_{1}, \ldots, c_{q}, \frac{1}{2} m-\frac{1}{2} n+1 ; 2 y^{2}
\end{array}\right]
\end{aligned}
$$

follows provided that the integral is convergent and term-by-term integration is permissible. A great many interesting particular cases of (2) are easily deducible: the following will be used in this paper.

$\int_{0}^{\infty} \sqrt{x y} x^{s} e^{-\xi x^{2}} H_{s+\frac{1}{2}}(x y) D_{2 s+2}(x) d x=(-1)^{s} y^{s+1} e^{-\xi y^{2}} D_{2 \varepsilon+1}(y)$

$\int_{0}^{\infty} \sqrt{x y} x^{s+\xi} e^{-\frac{k}{z} x^{2}} J_{s}(x y) D_{2 s+2}(x) d x=(-1)^{s+1} y^{s+t} e^{-k y^{2}} D_{2 s+2}(y)$

$\int_{0}^{\infty} e^{-i x^{2}} D_{2 s}(x) \cos x y d x=\sqrt{\frac{\pi}{2}}(-1)^{\circ} y^{2 s} e^{-t v^{2}}$

(4) is already known. ${ }^{(2)}$ Here and later $s$ is a non-negative integer.

2. The following integrals are obtained by integration with respect to $y$ from 0 to $\infty$ and inversion of the order of integrations.

Let us divide both sides of (5) by $\left(y^{2}+z^{2}\right)^{2}$, integrate, and use

$$
\int_{0}^{\infty} t^{2 s}\left(t^{2}+a^{2}\right)^{-s} e^{-\frac{1}{2} t^{2}} d t=2^{s-t} \Gamma\left(s+\frac{1}{2}\right) e^{j a^{2}} D_{-2 s}(a) .
$$

We get for $s>0$

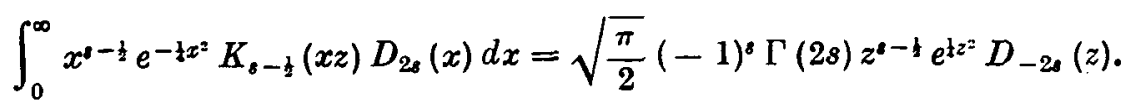


Multiplying (4) by $K_{s+\frac{1}{t}}(y z)$ and integrating $\int_{0}^{\infty} x^{2 s+1}\left(x^{2}+z^{2}\right)^{-\frac{1}{2}} e^{-i x^{2}} D_{2 s+2}(x) d x=\Gamma(2 s+2) z^{2 s+1} e^{t z^{2}} D_{-2 s-2}(z)$. (8)

Multiplying (5) by $e^{-y z}$, integrating, and using a well-known integral representation ${ }^{(3)}$ of parabolic cylinder functions we obtain ${ }^{(1)}$ $\int_{0}^{\infty} z\left(x^{2}+z^{2}\right)^{-1} e^{-\xi x^{2}} D_{2 s}(x) d x=\sqrt{\frac{\pi}{2}} \Gamma(2 s+1)(-1)^{s} e^{\natural z^{2}} D_{-2 s-1}(z) .(9)$ From this formula it follows that

$\int_{0}^{\infty} x^{2 s}\left(x^{2}+z^{2}\right)^{-1} e^{-\frac{1}{4} x^{2}} D_{2 s}(x) d x=\sqrt{\frac{\pi}{2}} \Gamma(2 s+1) z^{28-1} e^{\ddagger z^{2}} D_{-2 s-1}(z)$,

since $x^{28} /\left(x^{2}+z^{2}\right)=(-)^{8} z^{28} /\left(x^{2}+z^{2}\right)+$ an even polynomial of degree $2 s-2$ in $x$, and the contribution of that polynomial vanishes on account of the orthogonal property of parabolic cylinder functions.

Multiplying (3) by y $K_{8+\frac{1}{2}}(y z)$, integrating, and using (10) and the known result $\mathbf{t}^{(4)}$

we obtain

$$
\int_{0}^{\infty} x \mathbf{H}_{s+\frac{1}{2}}(x z) K_{s+1}(x y) d x=z^{8+3} y^{-s-1}\left(y^{2}+z^{2}\right)^{-1}
$$

$\int_{0}^{\infty} x^{s+\frac{s}{2}} e^{-\frac{1}{x^{2}} K_{s+\frac{1}{2}}}(x y) D_{2 s+1}(x) d x=\sqrt{\frac{\pi}{2}}(-1)^{s} \Gamma(2 s+3) y^{8-\frac{1}{2}} e^{\dagger y^{2}} D_{-28-3}(y)$.

3. To conclude this paper, a few integrals will be evaluated with the help of the operational calculus.

We write

$$
f(p) \doteqdot h(t)
$$

if

$$
f(p)=p \int_{0}^{\infty} e^{-p t} h(t) d t .
$$

The following results are well known ${ }^{(5)}$ :-

$$
\begin{aligned}
& f\left(\frac{p}{x}\right) \div h(x t) \quad(12) \quad p\left(p^{2}+x^{2}\right)^{-\frac{1}{2}} \div J_{0}(x t) \\
& p I_{s}(y \sqrt{p}) K_{s}(x \sqrt{p}) \doteqdot \frac{1}{2 t} \exp \left(-\frac{x^{2}+y^{2}}{4 t}\right) I_{s}\left(\frac{x y}{2 t}\right) \\
& \exp \left(-\frac{x^{2}+y^{2}}{4 p}\right) I_{s}\left(\frac{x y}{2 p}\right) \doteqdot J_{s}(x \sqrt{t}) J_{s}(y \sqrt{t}) \\
& \Gamma(s) p^{s+1} e^{\frac{4}{4} p^{2}} D_{-s}(p) \doteqdot \frac{d^{8}}{d t^{s}}\left(t^{8-1} e^{-\frac{1}{t^{3}}}\right), \quad(s>0) .
\end{aligned}
$$

Goldstein ${ }^{(\theta)}$ has proved that if $\phi(p) \div f(t)$ and $\psi(p) \doteqdot g(t)$ then

$$
\int_{0}^{\infty} \phi(t) g(t) t^{-1} d t=\int_{0}^{\infty} f(t) \psi(t) t^{-1} d t .
$$


In (8) let us put $z=p$, multiply by $p$, and interpret by means of (13) and (16), thus obtaining

$$
\int_{0}^{\infty} x^{28+1} e^{-\frac{1}{2} x^{2}} J_{0}(x z) D_{2 s+2}(x) d x=\frac{d^{2 s+1}}{d z^{2 \varepsilon+1}}\left(z^{28+1} e^{-\frac{1}{2} z^{2}}\right) .
$$

Apply (17) to (14) and (15) to find

$$
\begin{gathered}
\int_{0}^{\infty} I_{s}(z \sqrt{t}) J_{s}(y \sqrt{t}) J_{8}(z \sqrt{t}) K_{s}(y \sqrt{t}) d t \\
=\frac{1}{2} \int_{0}^{\infty} e^{-\frac{t}{2}\left(y^{2}+z^{2}\right) t} I_{g}^{2}\left(\frac{1}{2} y z t\right) d t=\frac{(-1)^{-8-\frac{1}{2}}}{\pi y z} Q_{8-\frac{1}{3}}\left(-\frac{y^{4}+z^{4}}{2 y^{2} z^{2}}\right) \\
(y \pm z>0) .
\end{gathered}
$$

In the integral

$$
\int_{0}^{\infty} x\left(x^{4}+4 k^{4}\right)^{-\frac{1}{2}} J_{0}(x y) d x=J_{0}(k y) K_{0}(k y)
$$

we put $k=\sqrt{\frac{1}{2} p}$, multiply by $p$, and have on interpretation

$$
p J_{0}\left(y \sqrt{\frac{1}{2} p}\right) K_{0}\left(y \sqrt{\frac{1}{2} p}\right) \div(2 t)^{-1} J_{0}\left(\frac{y^{2}}{4 t}\right) \text {. }
$$

Combining this with $p /(p+q) \doteqdot e^{-t}$ in the manner of (17),

$$
\int_{0}^{\infty} x e^{-2 x^{2}} J_{0}(x y) K_{0}(x y) d x=\frac{\pi}{16}\left\{\mathbf{H}_{0}\left(\frac{1}{4} y^{2}\right)-Y_{0}\left(\frac{1}{4} y^{2}\right)\right\}
$$

can be derived.

From (15) and (20) we deduce that

$$
\begin{aligned}
& \int_{0}^{\infty} J_{0}(x \sqrt{t}) J_{0}(y \sqrt{t}) J_{0}(z \sqrt{t}) K_{0}(z \sqrt{t}) d t \\
& =\frac{1}{2} \int_{0}^{\infty} e^{-t\left(x^{2}+y^{2}\right) t} J_{0}\left(\frac{1}{2} z^{2} t\right) I_{0}\left(\frac{1}{2} x y t\right) d t=\pi^{-1}\left(x y z^{2} i\right)^{-\frac{1}{2}} Q_{-\frac{1}{2}}\left(\frac{x^{4}+y^{4}-2 x^{2} y^{2}+4 z^{4}}{8 x y z^{2} i}\right) .
\end{aligned}
$$

I am indebted to a referee for valuable criticism and assistance in the preparation of this note.

\section{REFERENCES.}

(1) E. T. Whittaker and G. N. Watson, Modern Analysis, 3rd Edition, p. 353.

(2) C. S. Mitra, Math. Zeitschr., 43 (1943), p. 205.

(3) E. T. Whittaker and G. N. Watson, ibidem, p. 349.

(4) B. Mohan, Quart. J. of Math., 13 (1942), p. 40.

(5) H. S. Carslaw and J. C. Jaeger, Operational Methods in Applied Mathematics, p. xiv.

G. N. Watson, Bessel Functions, p. 395.

R. S. Varma, Proc. Cambridge Phil. Soc., 32 (1937), p. 210.

(6) S. Goldstein, Proc. London Math. Soc. (2), 34 (1932), p. 103.

Dacca University,

IN DIA. 